\title{
Mass spectrometry identification of granins and other proteins secreted by neuroblastoma cells
}

\author{
Wojciech Rozek • Malgorzata Kwasnik • Janusz Debski • \\ Jan F. Zmudzinski \\ Received: 7 December 2012 / Accepted: 24 February 2013 /Published online: 22 March 2013 \\ (C) The Author(s) 2013. This article is published with open access at Springerlink.com
}

\begin{abstract}
We used mass spectrometry-based protein identification to determine the presence of granins and other proteins in the mouse neuroblastoma secretome. We detected polypeptides derived from four members of the granin family: chromogranin A, chromogranin B, secretogranin III, and VGF. Many of them are derived from previously described biologically active regions; however, for VGF and $\mathrm{CgB}$, we detected peptides not related to known bioactivities. Along with granins, we identified 115 other proteins secreted by mouse neuroblastoma cells, belonging to different functional categories. Fifty-six out of 119 detected proteins possess the signal fragments required for translocation into endoplasmic reticulum. Sequences of remaining 63 proteins were analyzed using SecretomeP algorithm to determine probability of nonclassical secretion. Identified proteins are involved in the regulation of cell cycle, proliferation, apoptosis, angiogenesis, proteolysis, and cell adhesion.
\end{abstract}

Keywords Granins · Neuroblastoma $\cdot$ Secretome ·

Chromogranin $\cdot$ Secretogranin

\section{Introduction}

Over the last few years, there has been a growing interest in the study of cancer secretome comprising all the proteins that can be identified in the intestinal fluid of the tumor mass in vivo which play a key role in the signaling, communication, and migration of cells $[24,33]$. The term of "secretome" was introduced by Tjalsma in genome-based

W. Rozek $(\bowtie) \cdot$ M. Kwasnik · J. F. Zmudzinski

Department of Virology, National Veterinary Research Institute,

Al Partyzantow 57, 24-100 Pulawy, Poland

e-mail: wojciech.rozek@piwet.pulawy.pl

J. Debski

Mass Spectrometry Laboratory, Institute of Biochemistry

and Biophysics, PAS, Warsaw, Poland studies of Bacillus subtilis proteins [51]. Currently, the secretome studies include the proteins secreted via classical and nonclassical pathways but also shed from the surface of living cells [33]. The cell culture secretome can also be a suitable tool for investigating proteins released in vivo by tumors and used to identify putative tumor markers [9]. Neuroblastoma is the most common extracranial solid tumor of the sympathetic nervous system occurring in childhood. This neuroendocrine tumor secretes a range of proteins, which could serve as the potential biomarkers for diagnosis and monitoring of the treatment or disease progression [11, 46]. Several serum prognostic factors, such as neuron specific enolase, ferritin, and chromogranin $\mathrm{A}(\mathrm{CgA})$ have been used to predict neuroblastoma progression. $\mathrm{CgA}$ is currently the best available biomarker for the diagnosis of neuroendocrine tumors $[17,22,55]$. The granin family comprises nine members including $\mathrm{CgA}$ and $\mathrm{CgB}$, secretogranin $(\mathrm{Sg})$ II, III, IV (HISL-19), V (7B2), VI (NESP55), VII (VGF), and proSAAS $[15,16,18,56]$. Potential utility of $\mathrm{CgB}$, SgII, and VGF nerve growth factor-inducible protein (VGF) as biomarkers of neurological and psychiatric disorders has been described [6]. The expression patterns of granin-derived peptides seem to play an important role in differentiating between some benign and malignant neuroendocrine tumor types [39]. Granins are the main soluble proteins found in many neuroendocrine cells and in some neurons. They are present in large dense-core secretory vesicles and secreted during regulated exocytosis. Granins regulate the storage of catecholamines and ATP, exhibit $\mathrm{pH}-$ buffering capacities and thus they help to concentrate soluble products for secretion [7, 18, 32]. Their sequences contain pairs of basic amino acids and monobasic residues that are the potential cleavage sites for proteases. The granin-derived peptides fulfill autocrine and paracrine hormonal activities. Their relative abundance, functional significance, and secretion into the CSF or saliva and the general circulation made granin peptides tractable targets as biomarkers for many diseases of neuronal and endocrine 
origin [6]. We used mass spectrometry-based protein identification to determine the presence of the granin and other protein-derived peptides in the neuroblastoma secretome. This approach could deliver new information regarding neuroblastoma metabolism and new potential biomarkers of the disease.

\section{Material and methods}

\section{Sample preparation}

The mouse neuroblastoma cell line NEURO-2A was cultured in Eagle's medium with $10 \%$ fetal bovine serum. Oneday-old cultures were washed twice with PBS and the serum-free medium was applied. After $24 \mathrm{~h}$ culture, media were collected and centrifuged at $3,000 \times \mathrm{g}$ for $30 \mathrm{~min}$. The supernatants were concentrated on centrifugal filters with the molecular weight cutoff of $3 \mathrm{kDa}$ (Millipore, UFC900324). Proteins were precipitated using 5 volumes of cold acetone $\left(-20^{\circ} \mathrm{C}\right)$ and samples were centrifuged at $12,000 \times g$ for $10 \mathrm{~min}$ at $4{ }^{\circ} \mathrm{C}$. Subsequently, pellets were resuspended in $8 \mathrm{M}$ urea and diluted with $25 \mathrm{mM}$ ammonium bicarbonate. Proteins were reduced with $10 \mathrm{mM}$ DTT for $30 \mathrm{~min}$ at $57{ }^{\circ} \mathrm{C}$ and alkylated with $50 \mathrm{mM}$ iodoacetamide for $45 \mathrm{~min}$ at room temperature (RT) in a dark. Then samples were treated with $50 \mathrm{mM}$ DTT for $45 \mathrm{~min}$ at RT. Seventy micrograms of protein was used for tryptic digestion and protein identification. Solubilized proteins were digested overnight with sequencing grade modified trypsin (Promega, V5111, $0.01 \mu \mathrm{g}$ per $1 \mu \mathrm{g}$ of protein) and the reaction was quenched by adding $0.01 \%$ trifluoroacetic acid.

Mass spectrometry and data analysis

Digested peptides were applied to a RP-18 trapping column (nanoACQUITY UPLC Symmetry C18 Trap, Waters) using $0.1 \%$ trifluoroacetic acid mobile phase, and then transferred on to a HPLC RP-18 column (nanoACQUITY UPLC BEH C18 Column, Waters) using an acetonitrile gradient (0-30 \% in $0.1 \%$ formic acid) for $150 \mathrm{~min}$ at a flow rate of $200 \mathrm{~nL} / \mathrm{min}$. The column outlet was directly coupled to the ion source of the Ion Cyclotron Resonance spectrometer (LTQ61 FTICR, Thermo Electron). For protein identification, a series of three LC/MS runs were carried out on each sample, with the spectrometer running in data-dependent MS-to-MS/MS switch mode. Each run covered one of sectors of $m / z$ values: $300-600,500-800,700-2000$.

The parent and product ions lists for the database search were prepared by merging acquired raw files with Mascot Distiller software followed by Mascot Search Engine (Matrix Science, London, UK) against the NCBInr and IPI-
Mouse database. Search parameters for precursor and product ions mass tolerance were $30 \mathrm{ppm}$ and $0.8 \mathrm{Da}$, respectively. The other search parameters were as follows: enzyme specificity was set up to trypsin cleavage and variable modification of cysteine carbamidomethylation and methionine oxidation. Peptides with Mascot score exceeding the threshold value corresponding to $<5 \%$ false positive rate, calculated by Mascot procedure, were considered to be positively identified. At least two peptides per protein with score above the threshold were required for identification. The whole experiment was performed twice, using two biological replicates. Functional categorization of proteins was performed using Protein Analysis Through Evolutionary Relationship system (PANTHER, http:// www.pantherdb.org/) [36] and Gene Ontology (GO) classification [3]. We determined the presence of the signal peptides and the probability of non classical secretion using the UniProt database [2] and the SecretomeP algorithm [8], respectively.

\section{Electrophoresis and western blotting}

Electrophoresis and western blotting was done as it was described previously [29]. Twenty micrograms of proteins per line was used. The monoclonal antibodies for $\mathrm{CgA}$ (ChrA E-5), CgB (Chr-B N-20), SgIII (Sg III C-2), and VGF (H65) (Santa Cruz Biotechnology, INC) were used according to manufacturer recommendations.

\section{Results}

Our mass spectrometry analysis resulted in the identification of four members of the granin family in the pool of proteins secreted by mouse neuroblastoma cells: chromogranin A, chromogranin B, secretogranin III, and VGF nerve growth factor-inducible protein. The peptide sequences and identification parameters are presented in Table 1.

Chromogranin A was identified by detection of seven polypeptides (18\% sequence coverage). Five of them were homologous to the fragments of known biologically active peptides. One peptide (K.ELQDLALQGAK.E) was located within beta-granin/ vasostatin II region; two others (K.TEASEALPSEGK.G and K.DDGQSDSQAVDGD GK.T) were located in pancreastatin region of mouse chromogranin A. Another two polypeptides (K.VAHQ LQALR.R and R.AEDQELESLSAIEAELEK.V) were found in serpinin region. Positions of peptides identified within the protein sequence of chromogranin $\mathrm{A}$ are shown in Fig. 1.

We identified eight peptides of chromogranin B with sequence coverage of $16 \%$. Most of peptides detected for chromogranin $\mathrm{B}$ were localized within $\mathrm{N}$-terminal and in the 
Table 1 Granins identified in the secretome of mouse neuroblastoma cells

\begin{tabular}{|c|c|c|c|c|c|c|c|}
\hline $\begin{array}{l}\text { Protein name } \\
\text { (NCBI ID) }\end{array}$ & $\begin{array}{l}\text { Protein } \\
\text { score }\end{array}$ & $\begin{array}{l}\text { Sequence } \\
\text { coverage (\%) }\end{array}$ & $\begin{array}{l}\text { Observed } \\
\text { mass }\end{array}$ & $\begin{array}{l}\text { Calculated } \\
\text { mass }\end{array}$ & Start-end & $\begin{array}{l}\text { Peptide } \\
\text { score }\end{array}$ & Peptide sequence \\
\hline \multirow{7}{*}{$\begin{array}{l}\text { Chromogranin A } \\
\text { gi|6680932 }\end{array}$} & \multirow[t]{7}{*}{346} & \multirow[t]{7}{*}{18.1} & $1,034.5865$ & $1,034.5985$ & $453-461$ & 49.74 & K.VAHQLQALR.R \\
\hline & & & $1,044.434$ & $1,044.436$ & $374-382$ & 44.82 & R.LEGEDDPDR.S \\
\hline & & & $1,184.6366$ & $1,184.6401$ & $78-88$ & 36.95 & K.ELQDLALQGAK.E \\
\hline & & & $1,217.5751$ & $1,217.5775$ & $291-302$ & 44.05 & K.TEASEALPSEGK.G \\
\hline & & & $1,334.504$ & $1,334.5109$ & $339-348$ & 51.15 & K.QEEEEEEEER.L \\
\hline & & & $1,492.5831$ & $1,492.5914$ & $276-290$ & 90.83 & K.DDGQSDSQAVDGDGK.T \\
\hline & & & $2,003.0008$ & $2,002.9582$ & $435-452$ & 88.97 & R.AEDQELESLSAIEAELEK.V \\
\hline \multirow{10}{*}{$\begin{array}{l}\text { Chromogranin B } \\
\text { gi|6680934 }\end{array}$} & \multirow[t]{10}{*}{379} & \multirow[t]{10}{*}{16.2} & $1,114.4636$ & $1,114.4527$ & $399-407$ & 58.32 & R.HGEETEEER.S \\
\hline & & & $1,129.4734$ & $1,129.4822$ & $27-35$ & 36.48 & R.DHNEEMVTR.C \\
\hline & & & $1,197.6101$ & $1,197.6142$ & $438-447$ & 28.67 & R.LLDEGHYPVR.E \\
\hline & & & $1,258.5523$ & $1,258.5499$ & $216-226$ & 42.91 & R.ADAHSMELEEK.T \\
\hline & & & $1,274.5439$ & $1,274.5448$ & $216-226$ & 50.74 & R.ADAHSMELEEK.T (Ox. M) \\
\hline & & & $1,389.6124$ & $1,389.616$ & $300-311$ & 115.4 & K.SSYEGHPLSEER.R \\
\hline & & & $1,468.6466$ & $1,468.6542$ & $386-398$ & 42.24 & R.NHPDSELESTANR.H \\
\hline & & & $1,510.6417$ & $1,510.6059$ & $341-353$ & 48.53 & R.ASEEEPEYGEESR.S \\
\hline & & & $1,530.6815$ & $1,530.6798$ & $102-116$ & 60.17 & R.EDAGAPVEDSQGQTK.V \\
\hline & & & $1,986.9232$ & $1,986.9243$ & $131-148$ & 40.13 & R.EGVDDQESLRPSNQQASK.E \\
\hline $\begin{array}{l}\text { Secretogranin III } \\
\text { isoform } 1\end{array}$ & 497 & 23.1 & $1,473.7428$ & $1,473.7576$ & $233-246$ & 76.77 & K.VTPVAAVQDGFTNR.E \\
\hline \multirow[t]{5}{*}{ gi|6677867 } & & & $1,539.6937$ & $1,539.7205$ & $441-453$ & 74.49 & R.DFINQQADAYVEK.G \\
\hline & & & $1,765.802$ & $1,765.8118$ & $207-221$ & 40.88 & K.EANNYEETLDKPTSR.T \\
\hline & & & $1,875.9026$ & $1,875.8486$ & $99-115$ & 110.3 & R.SPPFDNQLNVEDADSTK.N \\
\hline & & & $2,734.49$ & $2,734.4388$ & $178-202$ & 143.1 & K.LLNLGLITESQAHTLEDEVAEALQK.L \\
\hline & & & $2,769.4311$ & $2,769.3596$ & $319-343$ & 95.21 & K.YGTISPEEGVSYLENLDETIALQTK.N \\
\hline $\begin{array}{l}\text { VGF nerve growth } \\
\text { factor }\end{array}$ & 469 & 13.1 & $1,009.4654$ & $1,009.4577$ & $423-431$ & 43.85 & R.SQEEAPGHR.R \\
\hline \multirow[t]{6}{*}{ gi|86476054 } & & & $1,113.5868$ & $1,113.5931$ & $509-519$ & 33.93 & R.SPQPPPPAPAR.D \\
\hline & & & $1,159.548$ & $1,159.5469$ & $353-361$ & 45.38 & R.ELQETQQER.E \\
\hline & & & $1,169.6189$ & $1,169.6193$ & $489-499$ & 36.03 & K.NAPPEPVPPPR.A \\
\hline & & & $1,172.5629$ & $1,172.5686$ & $577-586$ & 40.39 & R.HHPDLEAQAR.R \\
\hline & & & $1,381.7696$ & $1,381.7677$ & $299-311$ & 44.82 & R.LLQQGLAQVEAGR.R \\
\hline & & & $2,034.0237$ & $2,034.0521$ & $466-483$ & 83.11 & K.LHLPADDVVSIIEEVEEK.R \\
\hline
\end{tabular}

middle part of the protein. Two of them (R.DHNEE MVTR.C and R.LLDEGHYPVR.E) were located in $\mathrm{CgB}_{1-41}$ and GAWK regions.

We detected six different peptides of secretogranin III (sequence coverage $18 \%$ ). Three of them were related to

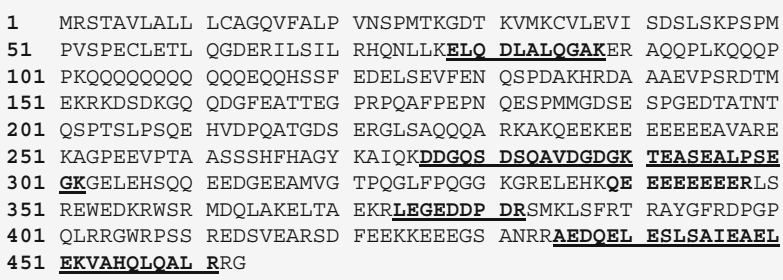

Fig. 1 Localization of identified peptides within chromogranin A sequence chromogranin A-binding domain (K.EANNYEETLD KPTSR.T, K.VTPVAAVQDGFTNR.E and K.YGTISPEE GVSYLENLDETIALQTK.N), two were mapped within the cholesterol-binding domain (R.SPPFDNQLN VEDADSTK.N and R.SPPFDNQLNVEDADSTK.N), and one within the carboxypeptidase $\mathrm{E}$ (CPE)-binding domain (R.DFINQQADAYVEK.G). For VGF, we detected seven peptides (13\% sequence coverage) including fragments homologous to NERP1-1 (R.LLQQGLAQVEAGR.R) and TLQP-62 (R.HHPDLEAQAR.R). We confirmed the presence of particular granins in neuroblastoma secretome using western blot. Proteins were detected with monoclonal antibodies at the level about $50-70 \mathrm{kDa}$ for secretogranin III, about $70 \mathrm{kDa}$ for chromogranin $\mathrm{B}$ and VGF, and $70-80 \mathrm{kDa}$ for chromogranin A (Fig. 2). 


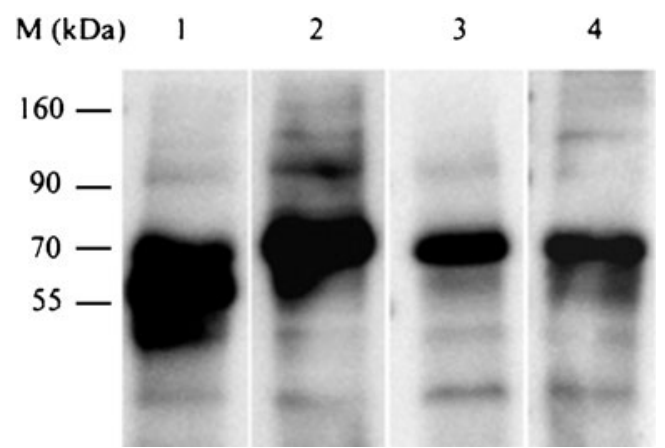

Fig. 2 Western blot detection of granins in the supernatants of neuroblastoma cells. Line 1 secretogranin III, line 2 chromogranin A, line 3 chromogranin B, line 4 VGF. $M$ molecular weight markers

Along with the four proteins belonging to the granins family, we identified 115 other proteins secreted by mouse neuroblastoma cells. Proteins were identified in two runs of identification with at least two peptides detected with the score above the threshold value. Five main molecular function categories of proteins were found by functional categorization using PANTHER system: catalytic (GO:0003824), binding (GO:0005488), structural molecule (GO:0005198), receptor (GO:0004872), and enzyme regulator (GO:0030 234) activities (Fig. 3).

All 119 detected proteins were verified for the presence of the signal fragments in their sequences. Fifty-six of them with signal sequences required for translocation into endoplasmic reticulum are presented in Table 2. The remaining 63 proteins without predicted signal sequence were analyzed using SecretomeP algorithm. We found out 17 proteins with neural network $(\mathrm{NN})$ score above 0.5 , which indicates that they could undergo nonclassical secretion (Table 3).

\section{Discussion}

In the pool of proteins secreted by mouse neuroblastoma cells, we identified four members of the granin family: $\mathrm{CgA}$, $\mathrm{CgB}$, Sg III, and VGF. The elevated level of $\mathrm{CgA}$ was previously found not only in the plasma of patients with neuroblastoma and ganglioneuroma but also with a wide range of tumors like pheochromocytoma; carcinoid tumors of the gastrointestinal tract, lung, and ovary; pancreatic endocrine tumors; and medullary thyroid carcinoma [11]. $\mathrm{CgA}$ may play a role in the regulation of tumor angiogenesis, vascular permeability, and endothelial barrier function affecting the response to certain therapies [30]. Human CgA-derived bioactive peptides involve vasostatin I, beta-granin/vasostatin II, prochromacin, chromacin, pancreastatin, catestatin, parastatin, WE-14, and serpinin $[1,12,19,28,34]$. We detected five peptides located within bioactive regions of $\mathrm{CgA}$ sequence: the peptide K.ELQDLALQGAK.E was located within beta-granin/ vasostatin II region, two others (K.TEASEALPSEGK.G and K.DDGQSDSQAVDGDGK.T) were located in pancreastatin region, and another two polypeptides (K.VAHQLQALR.R and R.AEDQELESLSAIEAELEK.V) were found in serpinin region. Increased level of pancreastatin concentrations correlates with tumor differentiation, localized clinical stage, and a favorable outcome for children with neuroblastoma. Kogner et al. suggested that pancreastatin in plasma and tumor tissue can be used as a marker indicating favorable tumor behavior [25]. The newly identified CgA-derived peptide - serpinin - stimulates transcription of protease nexin- 1 which is an inhibitor of plasmin protease and its increased expression stabilizes granule proteins in the Golgi complex [26]. Inhibition of plasmin
Fig. 3 Functional classification of proteins secreted by mouse neuroblastoma cells using the PANTHER analysis tool (www.pantherdb.org)

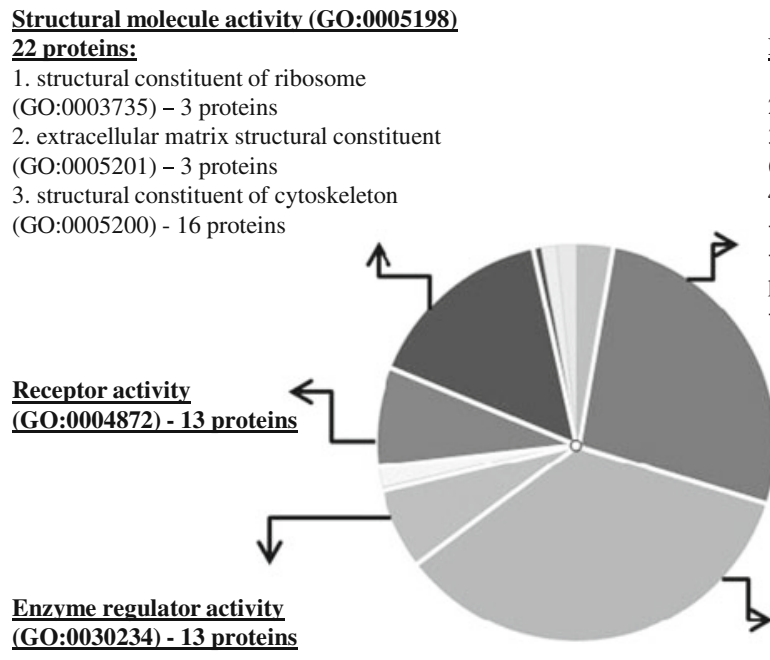

(GO:0030234) - 13 proteins
Binding (GO:0005488) - 51 proteins: 1. nucleic acid binding (GO:0003676) -18 proteins 2. calcium ion binding (GO:0005509) - 6 proteins 3. calcium-dependent phospholipid binding (GO:0005544) - 4 proteins

4. protein binding (GO:0005515) - 35 proteins: - receptor binding (GO:0005102) - 16 proteins - cytoskeletal protein binding (GO:0008092) - 4 proteins

- calmodulin binding (GO:0005516) - 5 proteins
Catalytic activity (GO:0003824) - 69 proteins: 1. ligase activity (GO:0016874) - 4 proteins 2. oxidoreductase activity (GO:0016491) -21 proteins 3. transferase activity (GO:0016740) - 15 proteins 4. hydrolase activity (GO:0016787) - 23 proteins 5. RNA splicing factor activity (GO:0031202) -7 proteins

6. lyase activity (GO:0016829) - 4 proteins

7. isomerase activity (GO:0016853) - 10 proteins 
Table 2 Proteins with the signal sequence identified in the secretome of neuroblastoma cells

\begin{tabular}{|c|c|c|c|c|c|}
\hline NCBI ID & Protein name & Gene & NCBI ID & Protein name & Gene \\
\hline gi|1083243 & Hypoxia upregulated protein 1 & Hyou1 & gi|1381582 & Sulfated glycoprotein 1 & Psap \\
\hline gi|11066226 & Cathepsin Z & Ctsz & gi|13938049 & Fibulin 1 & Fbln 1 \\
\hline gi|11596855 & Transferrin receptor protein 1 & Tfrc & gi|14250422 & Phosphogluconate dehydrogenase & Pgd \\
\hline gi|12746426 & CTF1-alpha & Clstn1 & gi|1568625 & Laminin subunit alpha-4 & Lama4 \\
\hline gi|12841873 & Nucleobindin-1 & Nucb1 & gi|192150 & Clusterin alpha chain & $\mathrm{Clu}$ \\
\hline gi|12860234 & Lysosomal protective prot.-cathepsin A & Ctsa & gi|2498391 & Follistatin-related protein 1 & Fstl1 \\
\hline gi|129729 & Protein disulfide-isomerase & $\mathrm{P} 4 \mathrm{hb}$ & gi|28972103 & Peroxidasin homolog & Pxdn \\
\hline gi|1345609 & Bone morphogenetic protein 1 & Bmp1 & gi|293691 & Laminin subunit gamma-1 & Lamc1 \\
\hline gi|17390745 & Complement $\mathrm{C} 1 \mathrm{~s}-\mathrm{A}$, light chain & $\mathrm{C} 1 \mathrm{sa}$ & gi|38372875 & Fibronectin & Fn1 \\
\hline gi|227293 & Cathepsin B & Ctsb & gi|396821 & Fibulin-1 & Fbln 1 \\
\hline gi|547841 & Low-density lipoprotein receptor & Ldlr & gi|437125 & Insulin-like growth factor-binding protein 5 & Igfbp5 \\
\hline gi|6678359 & Transketolase & Tkt & gi|50409 & Chromogranin B (Secretogranin-1) & Chgb \\
\hline gi|6753556 & Cathepsin D & Ctsd & gi|556299 & Collagen alpha-2(IV) chain & $\mathrm{Co} 14 \mathrm{a} 2$ \\
\hline gi|6755106 & Lysyl hydroxylase 1 & Plod1 & gi|607132 & Adipocyte enhancer-binding prot. 1 & Aebp1 \\
\hline gi|6755863 & Endoplasmin & Hsp90b1 & gi|6677867 & Secretogranin-3 & Scg3 \\
\hline gi|7242187 & Legumain & Lgmn & gi|6678077 & SPARC & Sparc \\
\hline gi|9558454 & Peptidase inhibitor 16 & Pi16 & gi|6680932 & Chromogranin A precursor & Chga \\
\hline gi|9790019 & Acid ceramidase subunit beta & Asah1 & gi $\mid 6755144$ & Galectin-3-binding protein & Lgals $3 b p$ \\
\hline gi|200397 & Protein disulfide-isomerase A3 & Pdia3 & gi|7657027 & Dickkopf-related protein 3 & Dkk3 \\
\hline gi|309085 & Amyloid beta & App & gi|86476054 & VGF nerve growth factor inducible & Vgf \\
\hline gi|1304157 & Heat shock $70 \mathrm{kDa}$ protein 5 & Hspa5 & gi|11762010 & Cystatin C precursor & Cst3 \\
\hline gi|114775 & Beta-2-microglobulin & $\mathrm{B} 2 \mathrm{~m}$ & gi|4959705 & Fibulin 2 & Fbln 2 \\
\hline gi|47894398 & Tropomyosin 4 & Tpm4 & gi|125490382 & Procollagen C-proteinase enhancer protein & Pcolce \\
\hline gi|148693781 & Neural cell adhesion molecule 1 & Ncam1 & gi|6753094 & Amyloid-like protein 2 & Aplp2 \\
\hline gi|53035 & Peptidyl-prolyl cis-trans isomerase & Ppib & gi|20381317 & Aggrecanase-1 & Adamts4 \\
\hline gi|6679465 & Glucosidase 2 subunit beta & Prkcsh & gi|6175081 & Fractalkine & $\mathrm{Cx} 3 \mathrm{cl} 1$ \\
\hline gi|10947006 & Fetuin-B & Fetub & gi|6680840 & Calumenin & Calu \\
\hline gi|12963609 & Sulfhydryl oxidase 1 & Qsox1 & gi|50852 & Granulin-7 & Grn \\
\hline
\end{tabular}

released during inflammatory process may also play a role in protecting cells under adverse pathophysiological conditions. Serpinin and its N-terminally modified form pyroglutamic-serpinin (pGlu-serpinin) also prevent reactive oxygen species and low potassium-induced cell death and hence they may be important in neuroprotection of the central nervous system, neurons, and pituitary cells. Neuroprotective effect of pGlu-serpinin involves activation of signal transduction pathway leading to upregulation of anti-apoptotic Bcl2 proteins [31]. Therefore, serpinins may play an important role in neuroblastoma progression through their anti-apoptotic activities preventing host mediated antitumor mechanisms.

SgIII consists of three functional regions: cholesterolbinding domain, CgA-binding domain, and membrane associated CPE-binding domain $[20,21]$. We detected six different peptides of SgIII (sequence coverage $18 \%$ ). Three of them were related to $\mathrm{CgA}$-binding domain (K.EANNYEETLDKPTSR.T, K.VTPVAAVQDGFTNR.E and K.YGTISPEEGVSYLENLDETIALQTK.N), two of them were mapped within the cholesterol-binding domain (R.SPPFDNQLNVEDADSTK.N and R.SPPFDNQLN VEDADSTK.N), and one within the CPE-binding domain (R.DFINQQADAYVEK.G). SgIII could mediate between the core aggregate and the cholesterol-rich secretory granule membrane, directing soluble binding cargo proteins to the secretory granules. Proteolytic fragments derived from SgIII have been described, however without specified biological activity [20, 21]. Expression of SgIII in 41 of 47 investigated neuroendocrine tumors was reported [38].

Immunoreactivity of VGF has been found in most welldifferentiated neuroendocrinal tumors [39, 41]. Neuroblastoma cells were identified as VGF positive and the VGF expression is upregulated during differentiation [43]. VGF peptides are present in endocrine cells early during development and adulthood and VGF increases in hyperplasia and tumors [41]. Different peptide fragments have been proposed to derive from VGF, including NAPP, NERP, 
Table 3 The NN scores rank of identified proteins without the signal sequence (scores calculated using SecretomeP algorithm)

\begin{tabular}{|c|c|c|c|c|c|c|c|}
\hline NCBI ID & Protein name & Gene & $\begin{array}{l}\mathrm{NN} \\
\text { score }\end{array}$ & NCBI ID & Protein name & Gene & $\begin{array}{l}\mathrm{NN} \\
\text { score }\end{array}$ \\
\hline gi|19111164 & $\begin{array}{l}\text { Small ubiquitin-related } \\
\text { modifier } 2\end{array}$ & Sumo2 & 0.883 & gi|12844989 & Phosphoglycerate mutase 1 & Pgam 1 & 0.408 \\
\hline gi|6679108 & Nucleophosmin & Npm1 & 0.803 & gi|193442 & Galectin-1 & Lgals1 & 0.402 \\
\hline gi|5007032 & Transgelin-2 & Tagln 2 & 0.790 & gi|202423 & Phosphoglycerate kinase 1 & Pgk1 & 0.400 \\
\hline gi|226471 & $\mathrm{Cu} / \mathrm{Zn}$ superoxide dismutase & Sod1 & 0.760 & gi|26324898 & Eukaryotic translation elongation factor 2 & Eef2 & 0.360 \\
\hline gi|1167510 & Ubiquitin & $\mathrm{Ubc}$ & 0.749 & gi|6671539 & Fructose-bisphosphate aldolase A & Aldoa & 0.357 \\
\hline gi|55291 & Vimentin & Vim & 0.728 & gi|387496 & Nucleoside diphosphate kinase A & Nme1 & 0.349 \\
\hline gi|6755911 & Thioredoxin & Txn & 0.698 & gi|3219774 & Peroxiredoxin-6 & $\operatorname{Prdx} 6$ & 0.346 \\
\hline gi|42542422 & $\begin{array}{l}\text { Heat shock cognate } 71 \mathrm{kDa} \\
\text { protein }\end{array}$ & Hspa8 & 0.641 & gi $\mid 5803225$ & 14-3-3 protein epsilon & Ywhae & 0.330 \\
\hline gi|13529464 & Nucleolin & $\mathrm{Ncl}$ & 0.570 & gi|45598372 & Brain acid soluble prot. 1 & Basp1 & 0.328 \\
\hline gi|6754524 & $\begin{array}{l}\text { L-lactate dehydrogenase A } \\
\text { chain }\end{array}$ & Ldha & 0.568 & gi|13569841 & Thioredoxin reductase 1 , cytoplasmic & Txnrd1 & 0.324 \\
\hline gi| 6755040 & Profilin-1 & Pfn1 & 0.560 & gi|51452 & $60 \mathrm{kDa}$ heat shock protein & Hspd1 & 0.320 \\
\hline gi|6678437 & $\begin{array}{l}\text { Translationally controlled } \\
\text { tumor protein }\end{array}$ & Tpt1 & 0.527 & gi| 4503545 & Eukaryotic transl. initiation factor $5 \mathrm{~A}-1$ & IF5A1 & 0.319 \\
\hline gi|1517864 & $\begin{array}{l}\text { Phosphatidylethanolamine } \\
\text { binding protein }\end{array}$ & Pebp1 & 0.522 & gi|6679078 & Nucleoside diphosphate kinase B & Nme2 & 0.307 \\
\hline gi|984938 & $\begin{array}{l}\text { Proteasome subunit beta } \\
\text { type- } 6\end{array}$ & Psmb6 & 0.512 & gi|3914804 & Heterogeneous nuclear rnp G & $\operatorname{Rbmx}$ & 0.300 \\
\hline gi|192050 & Aspartate aminotransferase & Got2 & 0.510 & gi|40556608 & Heat shock protein 1 beta & Hsp90ab1 & 0.296 \\
\hline gi|809561 & Actin, cytoplasmic 2 & Actg1 & 0.505 & gi|3065929 & 14-3-3 protein gamma & Ywhag & 0.290 \\
\hline gi|52865 & Lamin-A/C & Lmna & 0.505 & gi|20178336 & Tropomyosin alpha-3 & Tpm3 & 0.261 \\
\hline gi|74178273 & Actin, cytoplasmic 1 & Actb & 0.498 & gi|2495342 & Heat shock $70 \mathrm{kDa}$ protein 4 & Hspa4 & 0.261 \\
\hline gi|6754910 & $\begin{array}{l}\text { Nuclear migration protein } \\
\text { nudC }\end{array}$ & Nudc & 0.496 & gi|6756039 & $14-3-3$ protein theta & Ywhaq & 0.256 \\
\hline gi|7106387 & $\begin{array}{l}\text { Proteasome subunit alpha } \\
\text { type- } 5\end{array}$ & Psma5 & 0.494 & gi|115496850 & Spectrin alpha 2 & SPTA2 & 0.244 \\
\hline gi|576133 & $\begin{array}{l}\text { Glutathione } S \text {-transferase } \\
\text { P } 1\end{array}$ & Gstp1 & 0.485 & gi|1841387 & 14-3-3 protein zeta/delta & Ywhaz & 0.244 \\
\hline gi|202210 & Tubulin alpha-1B chain & Tubalb & 0.472 & gi|556301 & Elongation factor1-alpha1 & Eefla1 & 0.229 \\
\hline gi|2253159 & Peripherin & Prph & 0.461 & gi|790470 & Proliferation-associated protein 2 G4 & $\mathrm{Pa} 2 \mathrm{~g} 4$ & 0.194 \\
\hline gi|7106439 & Tubulin, beta 5 & Tubb5 & 0.458 & gi|13384620 & Heterogeneous nuclear rnp K & Hnrnpk & 0.177 \\
\hline gi|19527048 & $\begin{array}{l}\text { Heterogeneous nuclear } \\
\text { rnp F }\end{array}$ & Hnrnpf & 0.450 & gi|6754254 & Heat shock protein HSP 90 -alpha & Hsp90aa1 & 0.174 \\
\hline gi|387422 & Malate dehydrogenase & $\operatorname{Mdh} 2$ & 0.449 & gi|55217 & Transitional ER ATPase & Vcp & 0.163 \\
\hline gi|115558 & $\begin{array}{l}\text { Neural cell adhesion } \\
\text { molecule L1 }\end{array}$ & L1cam & 0.441 & gi|14389431 & Stress-induced phosphoprotein 1 & Stip1 & 0.155 \\
\hline gi|70794816 & Alpha-enolase & Eno1 & 0.439 & gi|2144100 & SET nuclear oncogene & Set & 0.103 \\
\hline gi|6679439 & $\begin{array}{l}\text { Peptidyl-prolyl cis-trans } \\
\text { isomerase A }\end{array}$ & Ppia & 0.421 & gi|1711240 & Heterogeneous nuclear rnp A1 & Hnrnpa1 & 0.087 \\
\hline gi|1405933 & $\begin{array}{l}\text { Pyruvate kinase isozymes } \\
\text { M1/M2 }\end{array}$ & Pkm2 & 0.418 & gi|3329498 & Heterogeneous nuclear rnp A2/B1 & Hnrnpa2b1 & 0.081 \\
\hline gi|1864018 & Triosephosphate isomerase & Tpi1 & 0.418 & gi|109866 & Nucleosome assembly protein 1 -like 1 & Nap111 & 0.042 \\
\hline gi|11230802 & Alpha-actinin-4 & Actn 4 & 0.417 & & & & \\
\hline
\end{tabular}

TLQP, AQEE, and LQEQ [40, 52, 53]. We detected seven peptides of VGF protein, four of them derived from previously described regions: R.LLQQGLAQVEAGR.R (NERP-1), K.NAPPEPVPPPR.A (NAPP-129), R.HHPDLE AQAR.R (TLQP-62/30), and R.SQEEAPGHR.R (APGH). Remaining three polypeptides (R.SPQPPPPAPAR.D ${ }_{(509-519)}$,
R.ELQETQQER.E(353-661), and K.LHLPADDVVSIIEE VEEK. $\left.\mathrm{R}_{(466-483)}\right)$ are derived from regions without known biological activity. Biological roles of VGF-derived peptides like regulation of energy balance, food intake, body fluid homeostasis, and reproduction were described $[4,5,13,23$, $45,52]$. Clinical and preclinical data links VGF-derived 
peptides in models of human depression (TLQP 62) [49], neuropathic and inflammatory pain (TLQP 21, AQEE 30) $[10,42]$, amyotrophic lateral sclerosis, Parkinson's, and Alzheimer's diseases [6, 37].

Different $\mathrm{CgB}$ peptides were detected in most neuroendocrine tumors [39]. Previously identified peptides derived from $\mathrm{CgB}$ include secretolytin ${ }_{(614-626)}$, $\operatorname{chrombacin}_{(564-626)}$ and fragment $\mathrm{CgB}_{(312-331)}, \mathrm{CgB}_{(1-41)}, \mathrm{GAWK}_{(420-493)}, \mathrm{CCB}$ (597-653), BAM1745(547-560), PE 11 $1_{(555-565)}$, Sr17(586-602), and $\mathrm{Hq}_{3} 4_{(603-636)}[27,48,54]$. We identified eight peptides of $\mathrm{CgB}$ in the pool of proteins secreted by mouse neuroblastoma cells. Most of the peptides detected in our study are localized in N-terminal part of chromogranin B sequence and are not homologous to known bioactive peptides derived from this protein. Two of them (R.DHNEEMVTR.C and R.LLDEGHYPVR.E) were located in regions previously described as $\mathrm{CgB}_{(1-41)}$ and GAWK. GAWK-like immunoreactivity is produced by a variety of endocrine tumors and may serve as a plasma tumor marker, especially in patients with pancreatic endocrine tumors [47]. Changes in the level of $\mathrm{CgB}$ and derived peptides in CSFs of patients with neurological diseases (e.g., multiple sclerosis, frontotemporal dementia, schizophrenia) were described (for review, [6]). Some conflicting results previously obtained from immunohistological studies using different antibodies may probably indicate differences in processing of particular epitopes in the tumors. That is why using alternative methods like mass spectrometry for investigating of peptides resulted from proteolytic cleavage of proteins ("degradomics") could overcome limitations of antibodybased methods [14].

Along with granins, we identified 115 other proteins secreted by mouse neuroblastoma cells. We examined their sequences for the presence of the extracellular transport signals to determine the mode of secretion. Granins and 52 other proteins with $\mathrm{N}$-terminal signal peptide included in Table 2 can be secreted via the classical pathway (translocation into endoplasmic reticulum, transport through Golgi complex, and secretory vesicles). Remaining 63 proteins could be transported using mechanisms of the nonclassical pathway (import into intracellular vesicles followed by its fusion with the plasma membrane, direct translocation across the plasma membrane, "flip-flop"-mediated secretion of membrane anchored proteins, or secretion in exosomes). To verify the possibility of nonclassical protein secretion, we applied SecretomeP algorithm [8] and we found 16 proteins with $\mathrm{NN}$ score above 0.5 (predictions of the non signal peptide triggered secretion, Table 3). However, NN score below 0.5 does not exclude that proteins can be secreted using the nonclassical pathway. So far, only limited number of proteins have been shown experimentally as nonclassical secretory proteins. We detected galectin and thioredoxin which have no signal peptides and were previously described as exported by the alternative pathway [35, 44]; however, NN score for galectin was calculated in the SecretomeP below 0.5 .

Functional categorization of detected proteins was performed using PANTHER system (http://www.pantherdb. org/) $[36,50]$. Proteins displayed five main molecular function categories: catalytic (GO:0003824), binding (GO:0005 488), structural molecule (GO:0005198), receptor (GO:00 04872), and enzyme regulator (GO:0030234) activities (Fig. 2). As expected for secreted proteins, a large group was classified as possessing receptor binding or receptor activities and catalytic or enzyme regulatory activities. We also indentified group of proteins with structural molecule activity (GO:0005198) belonging to the structural constituent of cytoskeleton (GO:0005200). The possible contribution of cytoskeletal proteins or their interactions seem to be a potential area for investigating cell communication mechanisms involved in neuroblastoma.

Proteins secreted by mouse neuroblastoma cells can represent different aspects of cancer pathobiology. Among them, we found proteins involved in cell cycle and proliferation (e.g., Nap111, Nudc, Vcp, Tubb5, Ywhae, Ywhaq, Ywhag, and Ywhaz), regulation of apoptosis (Clu, Set, Tpt1, Lgals1, Hspa4, and Hspa8), and angiogenesis (L1cam, Pcolce, and Col4A2), proteases, and proteases inhibitors (Ctsa, Ctsb, Ctsd, Ctsz, Cst3, Adamts4, Bmp1, Pcolce, and Lgmn). We found out also a group of proteins originated from cytoskeleton complex (Actg1, Actb, Tpm3, Tpm4, Prph, Vim, Nudc, Tubalb, and Tubb5) and proteins involved in the regulation of the cell adhesion (Ncam1, Ldlr, Fn1, L1cam, Fbln1, Lama4, Clstn1, and Lamc1). Proteins secreted by cancer cells might play an important role in cancer development and progression. Their analysis can provide insights into the metastasis, angiogenesis, tumor growth, and resistance to antiproliferative signals. Metastatic cascade involves epithelial-tomesenchymal transition, extracellular matrix degradation, intra- and extravasation, and anoikis evasion. All those steps require specific changes on the level of intra- and extracellular proteins (for review, [24]). Mass spectrometry-based approach to detect and characterize proteins of cancer secretome seems to be a valuable tool in analysis of biomarkers of oncological and neurological disorders. This approach can also supplement antibody-based methods when the protein is affected by differences in epitope processing in cells under pathological conditions. We detected polypeptides from four granins secreted by neuroblastoma cells. Many of them were homologous to previously described biologically active regions of granins; however, for VGF and $\mathrm{CgB}$, we detected peptides not related to known bioactivities. Identification of proteins in extracellular space, involved in cell cycle, proliferation, apoptosis, angiogenesis, proteolysis, and the cell adhesion, may help to explain mechanisms of cell communication during neuroblastoma progression. 
Acknowledgments This study was supported by National Science Centre, Poland (grant N N308 384935 to WR) and Centre for Preclinical Research and Technology CePT, Poland (POIG.02.02.0014-024/08-00 to JD).

\section{Conflicts of interest None}

Open Access This article is distributed under the terms of the Creative Commons Attribution License which permits any use, distribution, and reproduction in any medium, provided the original author(s) and the source are credited.

\section{References}

1. Aardal S, Helle KB. The vasoinhibitory activity of bovine chromogranin A fragment (vasostatin) and its independence of extracellular calcium in isolated segments of human blood vessels. Regul Pept. 1992;41(1):9-18.

2. Apweiler R, Bairoch A, Wu CH, Barker WC, Boeckmann B, Ferro S, Gasteiger E, Huang H, Lopez R, Magrane M, Martin MJ, Natale DA, O'Donovan C, Redaschi N, Yeh, LS (2004) UniProt: the Universal Protein knowledgebase. Nucleic Acids Res 32(Database issue): D115-9.

3. Ashburner M, Ball CA, Blake JA, Botstein D, Butler H, Cherry $\mathrm{JM}$, et al. Gene ontology: tool for the unification of biology. The Gene Ontology Consortium. Nat Genet. 2000;25(1):25-9.

4. Bartolomucci A, Bresciani E, Bulgarelli I, Rigamonti AE, Pascucci $\mathrm{T}$, Levi A, et al. Chronic intracerebroventricular injection of TLQP-21 prevents high fat diet induced weight gain in fast weight-gaining mice. Genes Nutr. 2009;4(1):49-57.

5. Bartolomucci A, La Corte G, Possenti R, Locatelli V, Rigamonti AE, Torsello A, et al. TLQP-21, a VGF-derived peptide, increases energy expenditure and prevents the early phase of diet-induced obesity. Proc Natl Acad Sci U S A. 2006;103(39):14584-9.

6. Bartolomucci A, Pasinetti GM, Salton SR. Granins as diseasebiomarkers: translational potential for psychiatric and neurological disorders. Neuroscience. 2010;170(1):289-97.

7. Bartolomucci A, Possenti R, Mahata SK, Fischer-Colbrie R, Loh YP, Salton SR. The extended granin family: structure, function, and biomedical implications. Endocr Rev. 2011;32(6):755-97.

8. Bendtsen JD, Jensen LJ, Blom N, Von Heijne G, Brunak S. Feature-based prediction of non-classical and leaderless protein secretion. Protein Eng Des Sel. 2004;17(4):349-56.

9. Chenau J, Michelland S, de Fraipont F, Josserand V, Coll JL, Favrot $\mathrm{MC}$, et al. The cell line secretome, a suitable tool for investigating proteins released in vivo by tumors: application to the study of p53-modulated proteins secreted in lung cancer cells. J Proteome Res. 2009;8(10):4579-91.

10. Cocco C, D'Amato F, Noli B, Ledda A, Brancia C, Bongioanni P, et al. Distribution of VGF peptides in the human cortex and their selective changes in Parkinson's and Alzheimer's diseases. J Anat. 2010;217(6):683-93.

11. Conlon JM. Granin-derived peptides as diagnostic and prognostic markers for endocrine tumors. Regul Pept. 2010;165(1):5-11.

12. Curry WJ, Johnston CF, Hutton JC, Arden SD, Rutherford NG, Shaw $\mathrm{C}$, et al. The tissue distribution of rat chromogranin Aderived peptides: evidence for differential tissue processing from sequence specific antisera. Histochemistry. 1991;96(6):531-8.

13. D'Amato F, Cocco C, Noli B, Cabras T, Messana I, Ferri GL. VGF peptides upon osmotic stimuli: changes in neuroendocrine regulatory peptides 1 and 2 in the hypothalamic-pituitary-axis and plasma. J Chem Neuroanat. 2012;44(2):57-65.
14. Doucet A, Butler GS, Rodriguez D, Prudova A, Overall CM. Metadegradomics: toward in vivo quantitative degradomics of proteolytic post-translational modifications of the cancer proteome. Mol Cell Proteomics. 2008;7(10):1925-51.

15. Fricker LD, McKinzie AA, Sun J, Curran E, Qian Y, Yan L, et al. Identification and characterization of proSAAS, a granin-like neuroendocrine peptide precursor that inhibits prohormone processing. J Neurosci. 2000;20(2):639-48.

16. Guerin M, Guillemot J, Thouennon E, Pierre A, El-Yamani FZ, Montero-Hadjadje M, et al. Granins and their derived peptides in normal and tumoral chromaffin tissue: implications for the diagnosis and prognosis of pheochromocytoma. Regul Pept. 2010;165(1):21-9.

17. Hann HW, Stahlhut MW, Evans AE. Serum ferritin as a prognostic indicator in neuroblastoma: biological effects of isoferritins. Prog Clin Biol Res. 1985;175:331-45.

18. Helle KB. The granin family of uniquely acidic proteins of the diffuse neuroendocrine system: comparative and functional aspects. Biol Rev Camb Philos Soc. 2004;79(4):769-94.

19. Helle KB. The chromogranin A-derived peptides vasostatin-I and catestatin as regulatory peptides for cardiovascular functions. Cardiovasc Res. 2010;85(1):9-16.

20. Hosaka M, Suda M, Sakai Y, Izumi T, Watanabe T, Takeuchi T. Secretogranin III binds to cholesterol in the secretory granule membrane as an adapter for chromogranin A. J Biol Chem. 2004;279(5):3627-34.

21. Hosaka M, Watanabe T, Sakai Y, Kato T, Takeuchi T. Interaction between secretogranin III and carboxypeptidase E facilitates prohormone sorting within secretory granules. J Cell Sci. 2005;118(Pt 20):4785-95.

22. Hsiao RJ, Seeger RC, Yu AL, O'Connor DT. Chromogranin A in children with neuroblastoma. Serum concentration parallels disease stage and predicts survival. J Clin Invest. 1990;85(5):1555-9.

23. Jethwa PH, Ebling FJ. Role of VGF-derived peptides in the control of food intake, body weight and reproduction. Neuroendocrinology. 2008;88(2):80-7.

24. Karagiannis GS, Pavlou MP, Diamandis EP. Cancer secretomics reveal pathophysiological pathways in cancer molecular oncology. Mol Oncol. 2010;4(6):496-510.

25. Kogner P, Bjellerup P, Svensson T, Theodorsson E. Pancreastatin immunoreactivity in favourable childhood neuroblastoma and ganglioneuroma. Eur J Cancer. 1995;31A(4):557-60.

26. Koshimizu H, Cawley NX, Kim T, Yergey AL, Loh YP. Serpinin: a novel chromogranin A-derived, secreted peptide up-regulates protease nexin-1 expression and granule biogenesis in endocrine cells. Mol Endocrinol. 2011;25(5):732-44.

27. Kroesen S, Marksteiner J, Leitner B, Hogue-Angeletti R, FischerColbrie R, Winkler H. Rat brain: distribution of immunoreactivity of PE-11, a peptide derived from chromogranin B. Eur J Neurosci. 1996;8(12):2679-89.

28. Kruger PG, Mahata SK, Helle KB. Catestatin (CgA344-364) stimulates rat mast cell release of histamine in a manner comparable to mastoparan and other cationic charged neuropeptides. Regul Pept. 2003;114(1):29-35.

29. Kwasnik M, Rozek W, Zmudzinski JF. Different patterns of multiplication of equine influenza virus serotypes H7N7 and H3N8 in chicken embryo. Bull Vet Inst Pulawy. 2011;55(4):575-9.

30. Loh YP, Cheng Y, Mahata SK, Corti A, Tota B. Chromogranin a and derived peptides in health and disease. J Mol Neurosci. 2012;48(2):347-56.

31. Loh YP, Koshimizu H, Cawley NX, Tota B. Serpinins: role in granule biogenesis, inhibition of cell death and cardiac function. Curr Med Chem. 2012;19(24):4086-92.

32. Machado JD, Diaz-Vera J, Dominguez N, Alvarez CM, Pardo MR, Borges R. Chromogranins A and B as regulators of vesicle cargo and exocytosis. Cell Mol Neurobiol. 2010;30(8):1181-7. 
33. Makridakis M, Vlahou A. Secretome proteomics for discovery of cancer biomarkers. J Proteomics. 2010;73(12):2291-305.

34. McVicar CM, Cunningham RT, Harriott P, Johnston CF, Buchanan KD, Curry WJ. Analysis of the post-translational processing of chromogranin $\mathrm{A}$ in rat neuroendocrine tissue employing an $\mathrm{N}$-terminal site-specific antiserum. J Neuroendocrinol. 2001;13(7):588-95.

35. Mehul B, Hughes RC. Plasma membrane targeting, vesicular budding and release of galectin 3 from the cytoplasm of mammalian cells during secretion. J Cell Sci. 1997;110(Pt 10):1169-78.

36. Mi H, Dong Q, Muruganujan A, Gaudet P, Lewis S, Thomas PD (2010) PANTHER version 7: improved phylogenetic trees, orthologs and collaboration with the Gene Ontology Consortium. Nucleic Acids Res 38(Database issue), D204-10.

37. Pasinetti GM, Ungar LH, Lange DJ, Yemul S, Deng H, Yuan X, et al. Identification of potential CSF biomarkers in ALS. Neurology. 2006;66(8):1218-22.

38. Portela-Gomes GM, Grimelius L, Stridsberg M. Secretogranin III in human neuroendocrine tumours: a comparative immunohistochemical study with chromogranins A and B and secretogranin II. Regul Pept. 2010;165(1):30-5.

39. Portela-Gomes GM, Grimelius L, Wilander E, Stridsberg M. Granins and granin-related peptides in neuroendocrine tumours. Regul Pept. 2010;165(1):12-20.

40. Possenti R, Rinaldi AM, Ferri GL, Borboni P, Trani E, Levi A. Expression, processing, and secretion of the neuroendocrine VGF peptides by INS-1 cells. Endocrinology. 1999;140(8):3727-35.

41. Rindi G, Licini L, Necchi V, Bottarelli L, Campanini N, Azzoni C, et al. Peptide products of the neurotrophin-inducible gene vgf are produced in human neuroendocrine cells from early development and increase in hyperplasia and neoplasia. J Clin Endocrinol Metab. 2007;92(7):2811-5.

42. Rizzi R, Bartolomucci A, Moles A, D'Amato F, Sacerdote P, Levi A, et al. The VGF-derived peptide TLQP-21: a new modulatory peptide for inflammatory pain. Neurosci Lett. 2008;441(1):129-33.

43. Rossi A, Granata F, Augusti-Tocco G, Canu N, Levi A, Possenti R. Expression in murine and human neuroblastoma cell lines of VGF, a tissue specific protein. Int J Dev Neurosci. 1992;10(6):527-34.

44. Rubartelli A, Bajetto A, Allavena G, Wollman E, Sitia R. Secretion of thioredoxin by normal and neoplastic cells through a leaderless secretory pathway. J Biol Chem. 1992;267(34):24161-4.

45. Salton SR, Ferri GL, Hahm S, Snyder SE, Wilson AJ, Possenti R, et al. VGF: a novel role for this neuronal and neuroendocrine polypeptide in the regulation of energy balance. Front Neuroendocrinol. 2000;21(3):199-219.

46. Sandoval JA, Hoelz DJ, Woodruff HA, Powell RL, Jay CL, Grosfeld JL, et al. Novel peptides secreted from human neuroblastoma: useful clinical tools? J Pediatr Surg. 2006;41(1):245-51.

47. Sekiya K, Ghatei MA, Salahuddin MJ, Bishop AE, Hamid QA, Ibayashi H, et al. Production of GAWK (chromograninB 420-493)-like immunoreactivity by endocrine tumors and its possible diagnostic value. J Clin Invest. 1989;83(6):183442.

48. Strub JM, Garcia-Sablone P, Lonning K, Taupenot L, Hubert P, Van Dorsselaer A, et al. Processing of chromogranin B in bovine adrenal medulla. Identification of secretolytin, the endogenous Cterminal fragment of residues 614-626 with antibacterial activity. Eur J Biochem. 1995;229(2):356-68.

49. Thakker-Varia S, Alder J. Neuropeptides in depression: role of VGF. Behav Brain Res. 2009;197(2):262-78.

50. Thomas PD, Campbell MJ, Kejariwal A, Mi H, Karlak B, Daverman R, et al. PANTHER: a library of protein families and subfamilies indexed by function. Genome Res. 2003;13(9):212941.

51. Tjalsma H, Bolhuis A, Jongbloed JD, Bron S, van Dijl JM. Signal peptide-dependent protein transport in Bacillus subtilis: a genomebased survey of the secretome. Microbiol Mol Biol Rev. 2000;64(3):515-47.

52. Toshinai K, Nakazato M. Neuroendocrine regulatory peptide-1 and -2 : novel bioactive peptides processed from VGF. Cell Mol Life Sci. 2009;66(11-12):1939-45.

53. Trani E, Ciotti T, Rinaldi AM, Canu N, Ferri GL, Levi A, et al. Tissue-specific processing of the neuroendocrine protein VGF. J Neurochem. 1995;65(6):2441-9.

54. Wang Z, Vandenberghe I, Depreitere J, Devreese B, Clerens S, Nouwen EJ, et al. Identification and characterization of novel chromogranin B-derived peptides from porcine chromaffin granules by liquid chromatography/electrospray tandem MS. Eur J Biochem. 2001;268(2):235-42.

55. Zeltzer PM, Marangos PJ, Evans AE, Schneider SL. Serum neuron-specific enolase in children with neuroblastoma. Relationship to stage and disease course. Cancer. 1986;57(6):1230-4.

56. Zhao E, Zhang D, Basak A, Trudeau VL. New insights into graninderived peptides: evolution and endocrine roles. Gen Comp Endocrinol. 2009;164(2-3):161-74. 